\title{
Untargeted Metabolomic Approach to Study the Serum Metabolites in Women with Polycystic Ovary Syndrome
}

\author{
Ying YU \\ Jiangsu University \\ PANLI TAN \\ Chinese Medicine Hospital of Zhejiang \\ ZHENCHAO ZHUANG \\ Chinese Medicine Hospital of Zhejiang \\ ZHEJIONG WANG \\ Chinese Medicine Hospital of Zhejiang \\ LINCHAO ZHU \\ Chinese Medicine Hospital of Zhejiang \\ RUYI QIU \\ Chinese Medicine Hospital of Zhejiang \\ HUAXI XU ( $\nabla$ xu_huaxi@163.com) \\ Jiangsu University
}

\section{Research Article}

Keywords: metabonomics analysis, UPLC-HRMS, polycystic ovary syndrome, serum metabolites

Posted Date: January 18th, 2021

DOI: https://doi.org/10.21203/rs.3.rs-144365/v1

License: (c) (i) This work is licensed under a Creative Commons Attribution 4.0 International License. Read Full License

Version of Record: A version of this preprint was published at BMC Medical Genomics on August 20th, 2021. See the published version at https://doi.org/10.1186/s12920-021-01058-y. 


\section{Abstract}

Background: Polycystic ovary syndrome (PCOS) is a kind of common endocrine syndrome as well as metabolic disorder, which harms the reproductive system and the overall body metabolism of the patients. This study, we aimed to investigate the difference of serum metabolic profiles of the patients with PCOS compared to the healthy controls.

Material and methods: a total of 62 study subjects including 31 PCOS patients and 31 matched healthy female controls were recruited, the clinical characteristics data were recorded at the time of recruitment, the laboratory biochemical data were detected. Then, we utilized metabolomics approach by UPLC-HRMS technology to study the metabolic changes between PCOS and controls.

Results: The metabolomics analysis showed that there were 68 were downregulated and 78 were upregulated metabolites in PCOS patients serum compared to the controls. These metabolites mainly belong to Triacylglycerols, Glycerophosphocholines, Acylcarnitines, Diacylglycerols, Peptides, Amino acids, Glycerophosphoethanolamines and fatty acid. Pathway analysis showed that these metabolites were enriched in pathways including Glycerophospholipid metabolism, Fatty acid degradation, Fatty acid biosynthesis, Ether lipid metabolism etc. Diagnosis value assessment by ROC analysis showed that the changed metabolites including Leu-Ala/Ile-Ala, 3-(4-Hydroxyphenyl) propionic acid, Ile-Val/Leu-Val, Gly-Val/Val-Gly, aspartic acid, DG(34:2)_DG(16:0/18:2), DG(34:1)_DG(16:0/18:1), Phe-Trp, DG(36:1)_DG(18:0/18:1), Leu-Leu/Leu-lle had higher AUC values, indicated significant role in PCOS.

Conclusion: The present study characterized the difference of metabolic and related pathway profiles in PCOS patients, this finding hope to provide potential metabolic marker for the prognosis and diagnosis of this disease.

\section{Background}

Around the world, approximately $15 \sim 20 \%$ of the women in childbearing age are affected with polycystic ovary syndrome (PCOS) according to the Rotterdam criteria [ ${ }^{1}$. This is one of the most common endocrine syndrome as well as metabolic disorder, which is mainly characterized with hyperandrogenism and insulin resistance (IR). The main manifestations of the PCOS patients are menstrual cycle irregular, oligo-ovulation and/or polycystic ovarian morphology, hyperandrogenism induced hirsutism and acne, and IR induced obesity [2]. But the diagnosis of PCOS is always a controversial issue and the criteria are continues to be updated [3-5]. Except for the impair on ovarian function and the overall body metabolism, the thereby caused anovulatory infertility and recurrent pregnancy loss also have tremendous harm to PCOS patients. In addition, due to the dysfunction of ovary and metabolism, long term consequences such as gynecological cancer, hypertension, atherosclerosis and type 2 diabetes mellitus (T2DM), cardiovascular disease (CVD) are also seems to have high incidences in PCOS women than normal populations [6-8]. In light of these risks, there is a strong need of finding reliable biochemical or molecular marker, which would enable to make the accurate diagnosis and effective therapy of PCOS.

Yet, we know surprising little about the exact mechanisms underlying the pathophysiology of PCOS, and this results in the limited available or effective therapies that ameliorate symptoms of PCOS or improve the thereby induced metabolic complications [9]. And shockingly, half of all women with PCOS are thought to remain undiagnosed. Genomic, proteomic, and metabolomic approaches to study the pathogenesis of diseases have been introduced to various diseases researching. Metabolomics involves the comprehensive characterization of metabolites and metabolism in biological systems, and is widely applied to better disease diagnose, understand the potential mechanisms, identify novel drug targets, customize drug treatments and monitor therapeutic outcomes [10]. Untargeted metabolomic approach, known as metabolic fingerprinting, mainly focuses on the identification and quantification of as many as possible low-molecular-weight compounds present in tested samples. This approach is commonly applied to uncover metabolic profiles, metabolic markers as well as to reveal new insights into the mechanisms underlying the pathogenesis of human diseases, including PCOS ${ }^{[} 11^{]}$.

In this study, based on metabolomics approach by utilizing an ultra-performance liquid chromatography-high resolution mass spectrometry (UPLC-HRMS) technology, we aim to characterize the metabolic fingerprints of the patients with PCOS and healthy controls, and also in hope of identifying potential metabolic marker for the prognosis and diagnosis of this disease.

\section{Material And Methods Study subjects}

All of the PCOS patients and healthy controls were recruited from the Zhejiang Provincial Hospital of Chinese Medicine (Hangzhou, China). This study was approved by the Ethics Committee of Zhejiang Provincial Hospital of Chinese Medicine. The signed informed consents were obtained from all the participators prior to inclusion in this study.

According to the Rotterdam criteria, 2003, PCOS patients can be diagnosed if two of the three criteria are present after excluding congenial adrenal hyperplasia, Cushing's syndrome, androgen secreting tumors, or other related disorders. The three criteria are (1) oligo- and/or anovulation; (2) clinical and/or biochemical signs of hyperandrogenism (clinical manifestations of hyperandrogenism include presence of acne, hirsutism, and androgenic alopecia); ( 3 ) polycystic ovaries by ultrasound examination: presence of 12 or more follicles in each ovary measuring $2-9 \mathrm{~mm}$ in diameter and/or ovarian volume $>10 \mathrm{~cm} 3$.

The inclusion criteria for PCOS cases in this study were: diagnosed with PCOS according to the Rotterdam criteria, 2003 [4]; adolescent females (18-40 years old); had at least 2 years of menstrual history. Exclusion criteria: had received any androgenic drug or sex steroid therapy in the past 3 months before the study; current pregnant, delivery or miscarriage within the preceding 3 months; congenital adrenal hyperplasia, androgen-secreting tumors, and other diseases with hyperandrogenism, thyroid dysfunction, hyperprolactinemia, cardiovascular diseases, diabetes or any chronic diseases. Controls group were healthy female volunteers, 18-40 years old, regular menstrual cycles and normal androgen levels, no PCOS and IR, and no evident disease was detected in them during 
the course of the study. According to the above mentioned inclusion/exclusion criteria, a total of 31 PCOS patients and 31 health participants were included from December of 2018 to April of 2019 in the present study.

The clinical characteristics data of the enrolled participators were recorded at the time of recruitment. After fasting for $8 \mathrm{~h}$, blood sample from each participator was collected. The serum samples were stored at $-80^{\circ} \mathrm{C}$ for subsequent assay.

\section{Clinical laboratory tests}

Serum concentrations of Fasting glucose, Fasting insulin, follicle-stimulating hormone (FSH), luteinizing hormone (LH), estradiol (E2), prolactin (PRL), testosterone $(T)$, progesterone (P), TC, TG, HDL-C, LDL-c in all PCOS patients and control participants were detected by Immulite 2000 analyzer (Siemens Healthcare Diagnostics Products Ltd., UK) using two site chemiluminescent immunometric assays.

\section{Sample preparation and metabolite extraction}

The polar metabolome extraction: After thawed at $4^{\circ} \mathrm{C}$, a $100 \mu \mathrm{L}$ serum samples were added with $400 \mu \mathrm{L}$ methanol-acetonitrile (1:1, v:v; including isotope internal standard tryptophan -d5, cetylic acid-[13C]12), centrifugated at 15,000 g for $15 \mathrm{~min}$. Then a $200 \mu \mathrm{L}$ supernatants were dried under low temperature vacuum (Thermo Scientific, USA) to obtained the sample for UPLC-HRMS analysis. Before analysis, the samples were redissolved with $100 \mu \mathrm{L} 10 \%$ methanol (including multiple internal standards).

The lipidomic metabolome extraction: After thawed at $4{ }^{\circ} \mathrm{C}$, a $50 \mu \mathrm{L}$ serum samples were added with $300 \mu \mathrm{L}$ methanol (including internal standards: Ceramide (d18:1/17:0) $\square \mathrm{PC}(17: 0 / 17: 0) \square T G(15: 0 / 15: 0 / 15: 0))$, swirled for $120 \mathrm{~s}$, and added with $900 \mu \mathrm{L}$ MTBE, $250 \mu \mathrm{L}$ ultrapure water. After vortex mixed and vibrated at room temperature for $15 \mathrm{~min}$, solution was placed under $4^{\circ} \mathrm{C}, 30 \mathrm{~min}$ for stratify. Then a $900 \mu \mathrm{L}$ supernatants were transferred into EP pipe and dried under low temperature vacuum (Thermo Scientific, USA) to obtained the sample for UPLC-HRMS analysis. Before analysis, the samples were redissolved with $600 \mu \mathrm{L}$ acetonitrile-isopropanol mixture.

\section{UPLC-HRMS instrumentation and measurement conditions}

Untargeted metabolomics analysis was conducted by using three different analytical methods (M1-3) on a Ultimate 3000 ultra-high performance liquid chromatograph coupled with Q ExactiveTM quadrupole-Orbitrap high resolution mass spectrometer (UPLC-HRMS) system (Thermo Scientific,USA).

\section{UPLC system}

Untargeted metabolomics analysis was conducted by using three different analytical methods (M1-3). Method 1 and 2 (M1, 2) was used for the polar metabolome extracts analysis on UPLC-HRMS system with positive and negative ionization detection, respectively. Metabolites were separated by an AcquityTM HSS C18 column (Waters Co., USA, $2.1 \times 100 \mathrm{~mm}$ ) for M1, and eluted by $0.1 \%$ formate/water (A) and acetonitrile (B) in linear gradient from $2 \%$ organic mobile phase to $98 \%$ in $10 \mathrm{~min}$. Furthermore, other mobile phases consisting of water and ammonium acetonitrile/methanol both containing ammonium bicarbonate buffer salt were employed to eluted metabolites separated on an AcquityTM BEH C18 column (Waters Co., USA, $1.7 \mu$ m, $2.1 \times 100$ $\mathrm{mm}$ ), the gradient was used as follow: from $0 \sim 10 \mathrm{~min}, 2 \%$ organic phase ramped to $100 \%$, and from 10 15 min, column washing and equilibrating. Untargeted lipidomic analysis was operated based on Method 3, the chromatographic separation conditions were maintained under positive and negative ionization detection mode, respectively. The used column was an Accucore C30 core-shell column, the mobile phase were $60 \%$ acetonitrile in water (A) and $10 \%$ acetonitrile in isopropanol (B) both containing $10 \mathrm{mM}$ ammonium formate and $0.1 \%$ formate. The separation gradient was optimized as follow: initial $10 \%$ B, ramping to $50 \%$ in $5 \mathrm{~min}$, and further increasing to $100 \%$ in $23 \mathrm{~min}$, the the rest $7 \mathrm{~min}$ for column washing and equilibration. For Method 1 3, the flow rate was $0.4 \mathrm{~mL} / \mathrm{min}$, injection volume was $5 \mu \mathrm{L}$, and column temperature was $50^{\circ} \mathrm{C}$.

\section{Mass spectrometer system}

For Method 1 2, the quadrupole-Orbitrap mass spectrometer was all operated under identical ionization parameters with a heated electrospray ionization source except ionization voltage including sheath gas $45 \mathrm{arb}$, aux gas 10 arb, heater temperature $355^{\circ} \mathrm{C}$, capillary temperature $320^{\circ} \mathrm{C}$ and S-Lens RF level $55 \%$. The metabolome extracts were profiled with full scan mode under 70,000 FWHM resolution with AGC 1 E6 and 200 ms max injection time. The scan range was 70 1000 m/z. QC samples were repeatedly injected to acquired Top 10 data dependent MS2 spectra (full scan-ddMS2) for comprehensive metabolite and lipid structural annotation. 17,500 FWHM resolution setting were used for full MS/MS data acquisition. Apex trigger, dynamic exclusion and isotope exclusion was turned on, precursor isolation window as set at 1.0 Da. Stepped normalized collision energy was employed for collision induced disassociation of metabolite using ultra-pure nitrogen as fragmentation gas. All the data acquired as centroid format. For Method 3 , the ionized lipid molecules were detected using the same parameters as previous description 6.3.1. 300-2000 m/z lipid extracts were profiled with the same parameters as the metabolome used. Lipid were structurally identified through acquiring data dependent MS2 spectra, the key settings included 70,000 FWHM full scan resolution, 17,500 FWHM MS/MS resolution, loop count 10, AGC target 3e6, maximum injection time 200 ms and 80 ms for full scan and MS/MS respectively, dynamic exclusion $8 \mathrm{~s}$. Stepped normalized collision energy $25 \%+40 \%$ and $35 \%$ were employed for positive and negative mode after optimization.

\section{Metabolomics data analysis}


The full scan and data-dependent MS2 metabolic profiles data were further processed with Compound Discoverer software for comprehensive component extraction. The polar metabolites were structural annotated through searching acquired MS2 against a local proprietary iPhenomeTM SMOL high resolution MS/MS spectrum library created using authentic standards, NIST 17 Tandem MS/MS library (National Institute of Standards and Technology), local version MoNA (MassBank of North America), as well as mzCloud library (Thermo Scientific, USA). Besides, exact m/z of MS1 spectra was searched against a local KEGG, HMDB metabolite chemical database. For metabolite identification or structural annotation, mass accuracy of precursor within $\pm 5 p p m$ was prerequisite, meanwhile, isotopic information including at least 1 isotopes within $10 \mathrm{ppm}$ and fit score of relative isotopic abundance pattern $70 \%$ were introduced to confirm the chemical formula in addition to exact mass. Furthermore, retention time information as well as high resolution MS/MS spectra similarity was employed to strictly confirm the structural annotation of metabolites. The area under curve (AUC) values as extracted as quantitative information of metabolites with XCalibur Quan Browser information, all peak areas data for the annotated metabolites were exported into Excel software for trim and organization before statistics (Microsoft, USA). And on the other hand, untargeted lipidomics data was processed with LipidSearch software including peak picking, lipid identification. The acquired MS2 spectra were searching against in silico predicted spectra of diverse phospholipid, neutral glycerolipid, spingolipid, neutral glycosphingolipids, glycosphingolipids, steroids, fatty ester, etc. The mass accuracy for precursor and MS/MS product ions searching were $5 \mathrm{ppm}$ and $5 \mathrm{mDa}$, respectively. The MS/MS similarity score threshold was set at 5 . The potential ionization adduct including hydrogen, sodium, ammonium for positive and hydrogen loss, formate and acetate adduct for negative mode. The lipid identification was strictly manually checked and investigated one-by-one to eliminate false positive chiefly basing on peak shake, adduct ions behavior, fragmentation pattern, and chromatographic behavior.

\section{Statistical analysis}

All the clinical data were computed using SPSS18.0 version software. An unpaired, two-tailed student $t$ test was performed on clinical biochemical data, the chi-square test was used for comparison of categorical variables. p-value $<0.05$ was considered to be statistically significant. The metabolome and lipidome data deriving from different measurements were normalized to sample weight used prior to further process, respectively. Then, the resultant quantitative information from foregoing methods were merged together and those detected with multiple methods was excluded to guaranteed uniqueness of metabolite and lipid, and then Log10 tranformed for final statistical analysis. The principal component analysis was conducted with SIMCA-P software (Umetrics, Sweden), and other univariate analysis including independent sample t-test and p value FDR adjust, as well as metabolic pathway analysis was conduct on MetaboAnalyst website.

\section{Results}

\section{Clinical characteristics and biochemical data of the study subjects}

The Clinical characteristics and biochemical data of the study subjects were collected and analyzed, and presented in Table 1. In this study, the study subjects included 31 healthy controls and 31 PCOS women. There is no statistically differences for the age, BMI between the two groups ( $p$ value >0.05). For biochemical data, the levels of fasting glucose, LH, T, TG, LDL-c and LH/FSH ratio were significantly higher in PCOS patients than those in controls, the levels of PRL, HDL-c were significantly lower in PCOS patients than those in controls ( $p$ value $<0.05)$.

Table 1

Clinical characteristic and biochemical data of the study subjects

\begin{tabular}{|llll|}
\hline & Control $(\mathbf{n = 3 1})$ & PCOS $(\mathbf{n = 3 1})$ & p-value \\
\hline Age [years] & $24.52 \pm 2.31$ & $24.20 \pm 4.49$ & 0.750 \\
\hline BMI [kg/m2] & $20.48 \pm 2.67$ & $22.27 \pm 3.56$ & 0.081 \\
\hline Fasting glucose [mmol/L] & $4.68 \pm 0.42$ & $5.25 \pm 1.20$ & $0.026<0.05$ \\
\hline Fasting insulin [pmol/L] & $6.9 \pm 3.38$ & $14.10 \pm 13.83$ & $0.007<0.05$ \\
\hline FSH [IU/L] & $5.15 \pm 1.32$ & $5.59 \pm 2.86$ & 0.440 \\
\hline LH [IU/L] & $5.85 \pm 2.74$ & $9.82 \pm 8.57$ & $0.017<0.05$ \\
\hline LH/FSH & $1.14 \pm 0.50$ & $1.73 \pm 0.97$ & $0.003<0.01$ \\
\hline PRL [mIU/L] & $466.83 \pm 231.05$ & $309.64 \pm 158.59$ & $0.003<0.01$ \\
\hline E2 [pmol/L] & $209.95 \pm 127.20$ & $220.25 \pm 246.80$ & 0.837 \\
\hline T [nmol/L] & $1.12 \pm 0.40$ & $1.65 \pm 0.67$ & $<0.001$ \\
\hline P [nmol/L] & $0.93 \pm 0.36$ & $1.05 \pm 0.80$ & 0.451 \\
\hline TC [mmol/L] & $4.44 \pm 0.63$ & $4.65 \pm 0.76$ & 0.306 \\
\hline TG [mmol/L] & $0.75 \pm 0.29$ & $1.13 \pm 0.51$ & $0.002<0.05$ \\
\hline HDL-c [mmol/L] & $2.23 \pm 0.52$ & $1.46 \pm 0.41$ & $<0.001$ \\
\hline LDL-c [mmol/L] & $1.69 \pm 0.29$ & $2.54 \pm 0.62$ & $<0.001$ \\
\hline
\end{tabular}




\section{Multivariate statistical analysis}

The multivariate statistical analysis were performed using SIMCAP software. The PCA (principal component analysis) analysis outlined the original distribution of metabolites in PCOS and control subjects. As shown in Fig. 1a, score plot of PCA suggested that there is no obvious outlier samples in two groups. The scatter plot classification in PCOS and control groups was observed in t[2] axis, but was failed to separated in t[1] axis. Hence, POLS-DA model was further applied. As the results indicated in Fig. 1b, PCOS samples could be clearly distinguished from the healthy control samples. The models possessed satisfactory fit of R2 $=0.93, Q 2=0.70$, which indicated the significant discrimination of the serum metabolomics signature between the two study groups. Figure $1 \mathrm{c}$ showed the result of permutation test made of OPLS-DA model, permutation plots of OPLS-DA model repeated 999 times verified the validation of the analysis. S plot of the OPLS-DA model indicated the influence of metabolite expression level on metabolic phenotype classification (Fig. 1d).

\section{Significant changed metabolotics identification by UPLC-HRMS}

The variables with FDR adjusted $\mathrm{p}$ value $<0.05$ was selected as remarkable significance in the OPLS-DA model. And these metabolotics were identified through the Compound Discoverer software for polar metabolites and LipidSearch software for lipidomics with the online database (like HMDB) or libraries. As a result, a total of 146 significantly changed metabolites were identified and selected as potential biomarkers of PCOS for subsequent analysis. Volcano plot showed that compared to the controls group, among these 146 significantly varied metabolites, 68 were downregulated, 78 were upregulated (Fig. 2a, Table 2). Heatmap of these 146 significantly changed metabolites in 31 PCOS samples and 31 control samples indicated that these metabolites have clustering correlativity in PCOS patients from healthy controls (Fig. 2b). After chemical structure classification of the identified 146 differential metabolites, Fig. 3 showed that the significantly changed metabolites mainly belonged to Triacylglycerol (36), Glycerophosphocholine (34), Acylcarnitine (15),

Diacylglycerol (15), Peptide (10), Amino acid (8), Glycerophosphoethanolamine (6), Fatty acid (FA) (3), etc. And top of the changed percentage metabolites (changed metabolites/total metabolites in this classification) were Diacylglycerol (78.95\%), Choline (50.00 \%), Acylcarnitine (48.39\%), Peptide (43.48 \%), Nucleoside\&nucleotide (27.27 \%), Glycerophosphocholine (21.12 \%), Neutral glycosphingolipid (18.18\%), Triacylglycerol (13.74 \%), etc. 
Table 2

The details about the differential metabolites identified in PCOS patients

\begin{tabular}{|c|c|c|c|c|c|}
\hline Class & Metabolite & HMDB ID & $\begin{array}{l}\text { Detection } \\
\text { Method }\end{array}$ & $\begin{array}{l}\text { Mass } \\
\text { accuracy } \\
\text { (PPM) }\end{array}$ & $\begin{array}{l}\mathrm{FD} \\
\text { ad } \\
\mathrm{p}-\mathrm{I}\end{array}$ \\
\hline Acylcarnitine & Palmitoylcarnitine(AcCa(16:0) & HMDB0000222 & M1 & -1.54 & $0 . C$ \\
\hline Acylcarnitine & Oleoylcarnitine(AcCa(18:1) & HMDB0005065 & M1 & -2.08 & $0 . C$ \\
\hline Acylcarnitine & Undecanoylcarnitine(AcCa(11:0) & HMDB0013321 & M1 & -1.68 & $0 . C$ \\
\hline Acylcarnitine & Isobutyry|-L-carnitine(AcCa(4:0) & HMDB0000736 & M1 & -0.47 & $0 . C$ \\
\hline Acylcarnitine & Tetradecanoylcarnitine(AcCa(14:0) & HMDB0005066 & M1 & -1.53 & $0 . c$ \\
\hline Acylcarnitine & Linoleyl carnitine(AcCa(18:2) & HMDB0006469 & M1 & -2.03 & $0 . c$ \\
\hline Acylcarnitine & Dodecenoylcarnitine(AcCa(12:1) & HMDB0013326 & M1 & -1.62 & $0 . c$ \\
\hline Acylcarnitine & 4-DecenoylcarnitineAcCa(10:1) & HMDB0013205 & M1 & -1.67 & $0 . C$ \\
\hline Acylcarnitine & Hexanoylcarnitine(AcCa(6:0) & HMDB0000705 & M1 & -0.55 & $0 . C$ \\
\hline Acylcarnitine & 9-Hexadecenoylcarnitine(AcCa(16:1) & HMDB0013207 & M1 & -1.33 & $0 . C$ \\
\hline Acylcarnitine & Tetradecenoylcarnitine(AcCa(14:1) & HMDB0002014 & M1 & -1.30 & $0 . c$ \\
\hline Acylcarnitine & Dodecanoylcarnitine(AcCa(12:0) & HMDB0002250 & M1 & -1.50 & $0 . C$ \\
\hline Acylcarnitine & Octanoylcarnitine( $\mathrm{AcCa}(8: 0)$ & HMDB0000791 & M1 & -1.52 & $0 . C$ \\
\hline Acylcarnitine & Tetradecadiencarnitine(AcCa(14:2) & HMDB0013331 & M1 & -1.49 & $0 . c$ \\
\hline Acylcarnitine & Decanoylcarnitine(AcCa(10:0) & HMDB0000651 & M1 & -1.09 & $0 . c$ \\
\hline Amino acid & Aspartic acid & HMDB0000191 & M2 & 3.33 & $0 . c$ \\
\hline Amino acid & L-Glutamic acid & HMDB0000148 & M2 & 3.78 & $0 . C$ \\
\hline Amino acid & L-Cystine & HMDB0000192 & M2 & 1.79 & $0 . c$ \\
\hline Amino acid & L-Phenylalanine & HMDB0000159 & M1 & -0.20 & $0 . c$ \\
\hline Amino acid & Acetylspermidine & HMDB0001276 & M1 & -0.92 & $0 . c$ \\
\hline Amino acid & Taurine & HMDB0000251 & M2 & 3.78 & $0 . C$ \\
\hline Amino acid & 3-(2-Hydroxyphenyl)propanoic acid & HMDB0033752 & M2 & 3.36 & $0 . C$ \\
\hline Amino acid & Proline betaine & HMDB0004827 & M1 & -0.08 & $0 . c$ \\
\hline Carbohydrate & Glucaric acid & HMDB0000663 & M2 & 2.69 & $0 . c$ \\
\hline Carbohydrate & L-Lactic acid & HMDB0000190 & M2 & 3.88 & $0 . c$ \\
\hline Cholesterol ester & $\operatorname{ChE}(16: 1) \_C h E(16: 1)$ & HMDB0000658 & M3 & -0.36 & $0 . C$ \\
\hline Choline & Glycerophosphocholine & HMDB0000086 & M1 & -0.92 & $0 . c$ \\
\hline Diacylglycerol & DG(34:2)_DG(16:0/18:2) & HMDB0007103 & M3 & 2.54 & $0 . c$ \\
\hline Diacylglycerol & DG(36:4)_DG(16:0/20:4) & HMDB0007113 & M3 & 2.35 & $0 . c$ \\
\hline Diacylglycerol & DG(34:1)_DG(16:0/18:1) & HMDB0007101 & M3 & 3.49 & $0 . C$ \\
\hline Diacylglycerol & DG(36:1)_DG(18:0/18:1) & HMDB0007159 & M3 & 2.88 & $0 . c$ \\
\hline Diacylglycerol & DG(34:3)_DG(16:1/18:2) & HMDB0007132 & M3 & 0.49 & $0 . c$ \\
\hline Diacylglycerol & DG(36:2)_DG(18:0/18:2) & HMDB0007161 & M3 & 1.49 & $0 . c$ \\
\hline Diacylglycerol & DG(34:2)_DG(16:1/18:1) & HMDB0007131 & M3 & 2.14 & $0 . c$ \\
\hline Diacylglycerol & DG(40:7)_DG(18:1/22:6) & HMDB0007208 & M3 & -0.89 & $0 . C$ \\
\hline Diacylglycerol & DG(38:4)_DG(18:1/20:3) & HMDB0007198 & M3 & 1.90 & $0 . c$ \\
\hline Diacylglycerol & DG(36:3)_DG(18:1/18:2) & HMDB0007219 & M3 & 2.40 & $0 . c$ \\
\hline Diacylglycerol & DG(36:4)_DG(18:2/18:2) & HMDB0007248 & M3 & -0.73 & $0 . c$ \\
\hline Diacylglycerol & DG(36:2)_DG(18:1/18:1) & HMDB0007218 & M3 & 3.21 & $0 . C$ \\
\hline Diacylglycerol & DG(40:6)_DG(18:1/22:5) & HMDB0007207 & M3 & 1.92 & $0 . c$ \\
\hline
\end{tabular}




\begin{tabular}{|c|c|c|c|c|c|}
\hline Class & Metabolite & HMDB ID & $\begin{array}{l}\text { Detection } \\
\text { Method }\end{array}$ & $\begin{array}{l}\text { Mass } \\
\text { accuracy } \\
\text { (PPM) }\end{array}$ & $\begin{array}{l}\text { FD } \\
\text { ad } \\
p-i\end{array}$ \\
\hline Diacylglycerol & DG(38:5)_DG(18:2/18:3) & HMDB0007112 & M3 & 1.76 & $0 . c$ \\
\hline Diacylglycerol & DG(38:4)_DG(18:0/20:4) & HMDB0007170 & M3 & 2.64 & $0 . c$ \\
\hline Fatty acid & Dihomo-alpha-linolenic acid (FFA(20:3n3) & HMDB0060039 & M2 & 1.69 & $0 . c$ \\
\hline Fatty acid & Myristoleic acid isomer2 & HMDB0002000 & M2 & 2.46 & $0 . \mathrm{C}$ \\
\hline Fatty acid & Myristoleic acid isomer 1 & HMDB0002000 & M2 & 2.46 & $0 . c$ \\
\hline Glycerophosphocholine & LysoPC(0-18:1/0:0) & LMGP01060039 & M1 & -0.46 & $0 . c$ \\
\hline Glycerophosphocholine & LysoPC(16:0e) & & M1 & -1.00 & $0 . c$ \\
\hline Glycerophosphocholine & LysoPC(0:0/18:0) & HMDB0011128 & M1 & -0.65 & $0 . \mathrm{C}$ \\
\hline Glycerophosphocholine & LysoPC(0-18:0/0:0) & HMDB0011149 & M1 & -0.63 & $0 . c$ \\
\hline Glycerophosphocholine & LysoPC(18:0/0:0) & HMDB0010384 & M1 & -0.42 & $0 . \mathrm{C}$ \\
\hline Glycerophosphocholine & LysoPC(P-18:1/0:0) & HMDB0010408 & M1 & -0.41 & $0 . c$ \\
\hline Glycerophosphocholine & LysoPC(P-16:0/0:0) & HMDB0010407 & M1 & -0.96 & $0 . c$ \\
\hline Glycerophosphocholine & LysoPC(19:0) & & M1 & -1.02 & $0 . c$ \\
\hline Glycerophosphocholine & LysoPC(20:1/0:0) & HMDB0010391 & M1 & -1.33 & $0 . c$ \\
\hline Glycerophosphocholine & PC(34:2)_PC(16:0/18:2) & HMDB0007973 & M3 & -0.45 & $0 . c$ \\
\hline Glycerophosphocholine & PC(32:0e)_PC(32:0e) & LMGP01020029 & M3 & 1.50 & $0 . \mathrm{C}$ \\
\hline Glycerophosphocholine & PC(37:2)_PC(37:2)mixture & HMDB0008592 & M3 & 2.08 & $0 . c$ \\
\hline Glycerophosphocholine & PC(32:0p)_PC(32:0p) & HMDB0011206 & M3 & 1.11 & $0 . c$ \\
\hline Glycerophosphocholine & $P C(36: 2 e) \_P C(36: 2 e)$ & HMDB0013418 & M3 & 2.44 & $0 . c$ \\
\hline Glycerophosphocholine & PC(36:1p)_PC(18:0p/18:1) & HMDB0008127 & M3 & 2.44 & $0 . \mathrm{C}$ \\
\hline Glycerophosphocholine & PC(34:0p)_PC(34:0p) & HMDB0011239 & M3 & 1.82 & $0 . c$ \\
\hline Glycerophosphocholine & $P C(38: 6 p) \_P C(38: 6 p)$ & HMDB0011229 & M3 & -1.62 & $0 . c$ \\
\hline Glycerophosphocholine & PC(34:2p)_PC(34:2p) & HMDB0011211 & M3 & 0.42 & $0 . c$ \\
\hline Glycerophosphocholine & $\mathrm{PC}(35: 2) \_P C(17: 0 / 18: 2)$ & LMGP01011505 & M3 & -2.68 & $0 . \mathrm{C}$ \\
\hline Glycerophosphocholine & PC(34:1p)_PC(34:1p) & HMDB0011210 & M3 & 1.37 & $0 . c$ \\
\hline Glycerophosphocholine & LysoPC(18:2/0:0) & HMDB0010386 & M1 & -0.44 & $0 . c$ \\
\hline Glycerophosphocholine & PC(33:2)_PC(33:2) & HMDB0007940 & M3 & -2.06 & $0 . \mathrm{C}$ \\
\hline Glycerophosphocholine & $P C(36: 3) \_P C(16: 0 / 20: 3)$ & HMDB0007980 & M3 & -2.02 & $0 . c$ \\
\hline Glycerophosphocholine & LysoPC(0:0/18:2) & HMDB0061700 & M1 & -0.44 & $0 . c$ \\
\hline Glycerophosphocholine & PC(42:3)_PC(18:1/24:2) & & M3 & 0.18 & $0 . c$ \\
\hline Glycerophosphocholine & PC(36:3)_PC(18:1/18:2) & HMDB0008105 & M3 & -2.02 & $0 . c$ \\
\hline Glycerophosphocholine & PC(32:1p)_PC(32:1p) & & M3 & -2.43 & $0 . c$ \\
\hline Glycerophosphocholine & $P C(40: 7 p) \_P C(40: 7 p)$ & HMDB0011295 & M3 & -2.19 & $0 . c$ \\
\hline Glycerophosphocholine & PC(33:0e)_PC(18:0e/15:0) & & M3 & 1.27 & $0 . c$ \\
\hline Glycerophosphocholine & PC(32:1e)_PC(32:1e) & HMDB0013404 & M3 & -0.67 & $0 . c$ \\
\hline Glycerophosphocholine & PC(35:2)_PC(35:2) & & M3 & 0.99 & $0 . \mathrm{C}$ \\
\hline Glycerophosphocholine & PC(35:3)_PC(35:3) & & M3 & -2.26 & $0 . c$ \\
\hline Glycerophosphocholine & $P C(30: 0 e) \_P C(30: 0 e)$ & HMDB0013341 & M3 & 1.32 & $0 . c$ \\
\hline Glycerophosphocholine & PC(33:0p)_PC(33:0p) & HMDB0011238 & M3 & 0.39 & $0 . c$ \\
\hline Glycerophosphoethanolamine & LysoPE(0:0/22:6) & HMDB0011496 & M1 & -0.76 & $0 . c$ \\
\hline Glycerophosphoethanolamine & LysoPE(0:0/18:2) & HMDB0011477 & M1 & -1.03 & $0 . c$ \\
\hline Glycerophosphoethanolamine & PE(40:5)_PE(18:1/22:4) & HMDB0009075 & M3 & -1.19 & $0 . c$ \\
\hline
\end{tabular}




\begin{tabular}{|c|c|c|c|c|c|}
\hline Class & Metabolite & HMDB ID & $\begin{array}{l}\text { Detection } \\
\text { Method }\end{array}$ & $\begin{array}{l}\text { Mass } \\
\text { accuracy } \\
\text { (PPM) }\end{array}$ & $\begin{array}{l}\text { FD } \\
\text { ad } \\
p-i\end{array}$ \\
\hline Glycerophosphoethanolamine & LysoPE(20:4/0:0) & HMDB0011517 & M1 & -1.41 & $0 . c$ \\
\hline Glycerophosphoethanolamine & LysoPE(22:6/0:0) & HMDB0011526 & M1 & -0.88 & $0 . c$ \\
\hline Glycerophosphoethanolamine & LysoPE(18:2/0:0) & HMDB0011507 & M1 & -0.84 & $0 . c$ \\
\hline Micorbial metabolites & 3-(4-Hydroxyphenyl)propionic acid(Desaminotyrosine) & HMDB0002199 & M2 & -0.05 & $0 . \mathrm{C}$ \\
\hline Neutral glycosphingolipid & CerG3GNAc1(d34:1)_Tetrahexosylceramide(d18:1/16:0) & HMDB0004960 & M3 & 1.29 & $0 . c$ \\
\hline Neutral glycosphingolipid & CerG2(d42:1)_LacCer(d18:1/24:0) & HMDB0011595 & M3 & 2.97 & $0 . c$ \\
\hline Neutral glycosphingolipid & CerG3(d42:2)_CerG3(d18:1/24:1) & HMDB0004883 & M3 & 3.52 & $0 . c$ \\
\hline Neutral glycosphingolipid & CerG3(d42:2)_CerG3(d42:2) & HMDB0004883 & M3 & 3.52 & $0 . \mathrm{C}$ \\
\hline Nucleoside\&nucleotide & Hypoxanthine & HMDB0000157 & M1 & 0.35 & $0 . c$ \\
\hline Nucleoside\&nucleotide & Xanthine & HMDB0000292 & M1 & -0.03 & $0 . \mathrm{C}$ \\
\hline Nucleoside\&nucleotide & Inosine & HMDB0000195 & M2 & 0.44 & $0 . c$ \\
\hline Organic acid & Glutaric acid isomers & HMDB0000661 & M2 & 3.91 & $0 . c$ \\
\hline Peptide & Gly-Val/Val-Gly & HMDB0028854/HMDB0029127 & M2 & 3.53 & $0 . c$ \\
\hline Peptide & Glp-Gly & HMDB0061890 & M2 & 3.62 & $0 . \mathrm{C}$ \\
\hline Peptide & Phe-Phe & HMDB0013302 & M2 & 2.02 & $0 . c$ \\
\hline Peptide & Phe-Trp & HMDB0029006 & M1 & -1.33 & $0 . c$ \\
\hline Peptide & Ser-Hyp/Hyp-Ser & HMDB0029040/HMDB0028872 & M1 & -0.35 & $0 . c$ \\
\hline Peptide & Glu-lle & HMDB0028822 & M1 & -0.82 & $0 . c$ \\
\hline Peptide & Glu-Gln & HMDB0028817 & M2 & 0.67 & $0 . c$ \\
\hline Peptide & Leu-Leu/Leu-lle & HMDB0028933 & M1 & -0.61 & $0 . \mathrm{C}$ \\
\hline Peptide & Ile-Val/Leu-Val & HMDB0028920/HMDB0028942 & M1 & -0.53 & $0 . c$ \\
\hline Peptide & Leu-Ala/Ile-Ala & HMDB0028922/HMDB0028900 & M1 & 0.09 & $0 . \mathrm{C}$ \\
\hline Sphingolipid & Sphingosine & HMDB0000252 & M1 & -1.44 & $0 . c$ \\
\hline Sphingolipid & Sphinganine 1-phosphate & HMDB0001383 & M1 & -1.74 & $0 . \mathrm{C}$ \\
\hline Triacylglycerol & TG(46:0)_TG(16:0/14:0/16:0) & HMDB0010411 & M3 & -1.43 & $0 . c$ \\
\hline Triacylglycerol & TG(48:0)_TG(16:0/16:0/16:0) & HMDB0005356 & M3 & -0.85 & $0 . c$ \\
\hline Triacylglycerol & TG(50:0)_TG(18:0/16:0/16:0) & HMDB0108576 & M3 & -0.59 & $0 . \mathrm{C}$ \\
\hline Triacylglycerol & TG(60:9)_TG(18:0/20:4/22:5) & HMDB0045200 & M3 & -0.79 & $0 . c$ \\
\hline Triacylglycerol & TG(52:1)_TG(18:0/16:0/18:1) & HMDB0010431 & M3 & 0.02 & $0 . c$ \\
\hline Triacylglycerol & TG(48:1)_TG(16:0/14:0/18:1) & HMDB0010414 & M3 & -1.12 & $0 . c$ \\
\hline Triacylglycerol & TG(54:6)_TG(16:0/16:0/22:6) & HMDB0044613 & M3 & -0.58 & $0 . c$ \\
\hline Triacylglycerol & TG(54:7)_TG(18:1/14:0/22:6) & HMDB0049719 & M3 & 0.61 & $0 . c$ \\
\hline Triacylglycerol & TG(56:6)_TG(18:0/16:0/22:6) & HMDB0044747 & M3 & -0.08 & $0 . \mathrm{C}$ \\
\hline Triacylglycerol & TG(52:6)_TG(14:0/16:0/22:6) & HMDB0042903 & M3 & 0.80 & $0 . c$ \\
\hline Triacylglycerol & TG(48:2)_TG(12:0/18:1/18:1) & LMGL03012670 & M3 & -1.31 & $0 . \mathrm{C}$ \\
\hline Triacylglycerol & TG(48:2)_TG(16:0/14:0/18:2) & HMDB0010415 & M3 & -1.31 & $0 . \mathrm{C}$ \\
\hline Triacylglycerol & TG(50:1)_TG(16:0/16:0/18:1) & HMDB0005360 & M3 & -0.42 & $0 . c$ \\
\hline Triacylglycerol & TG(51:1)_TG(16:0/17:0/18:1) & LMGL03010051 & M3 & 3.30 & $0 . c$ \\
\hline Triacylglycerol & TG(54:4)_TG(18:0/16:0/20:4) & HMDB0044738 & M3 & -0.97 & $0 . c$ \\
\hline Triacylglycerol & TG(52:2)_TG(18:0/16:0/18:2) & HMDB0044734 & M3 & -0.09 & $0 . c$ \\
\hline Triacylglycerol & TG(52:4)_TG(16:0/16:0/20:4) & HMDB0005363 & M3 & -0.31 & $0 . c$ \\
\hline Triacylglycerol & TG(54:1)_TG(18:0/18:0/18:1) & HMDB0005395 & M3 & 2.99 & $0 . c$ \\
\hline
\end{tabular}

Page 8/18 


\begin{tabular}{|c|c|c|c|c|c|}
\hline Class & Metabolite & HMDB ID & $\begin{array}{l}\text { Detection } \\
\text { Method }\end{array}$ & $\begin{array}{l}\text { Mass } \\
\text { accuracy } \\
\text { (PPM) }\end{array}$ & $\begin{array}{l}\text { FD } \\
\text { ad } \\
\text { p-i }\end{array}$ \\
\hline Triacylglycerol & TG(47:2)_TG(14:0/15:0/18:2) & HMDB0043227 & M3 & 0.36 & $0 . c$ \\
\hline Triacylglycerol & TG(51:0)_TG(18:0/16:0/17:0) & HMDB0108587 & M3 & 0.02 & $0 . c$ \\
\hline Triacylglycerol & TG(49:1)_TG(15:0/16:0/18:1) & HMDB0043027 & M3 & -0.54 & $0 . c$ \\
\hline Triacylglycerol & TG(49:1)_TG(49:1) & & M3 & -0.54 & $0 . c$ \\
\hline Triacylglycerol & TG(54:2)_TG(18:0/18:0/18:2) & HMDB0005397 & M3 & 2.83 & $0 . c$ \\
\hline Triacylglycerol & TG(56:4)_TG(18:0/18:0/20:4) & HMDB0044771 & M3 & -0.07 & $0 . c$ \\
\hline Triacylglycerol & TG(50:2)_TG(16:0/16:0/18:2) & HMDB0005362 & M3 & -1.69 & $0 . c$ \\
\hline Triacylglycerol & TG(52:0)_TG(18:0/16:0/18:0) & HMDB0044722 & M3 & 2.83 & $0 . c$ \\
\hline Triacylglycerol & TG(53:1)_TG(15:0/22:0/16:1) & HMDB0043112 & M3 & -0.49 & $0 . c$ \\
\hline Triacylglycerol & TG(53:1)_TG(17:0/18:0/18:1) & LMGL03010119 & M3 & -0.49 & $0 . c$ \\
\hline Triacylglycerol & TG(56:7)_TG(16:0/18:1/22:6) & HMDB0044135 & M3 & -0.72 & $0 . c$ \\
\hline Triacylglycerol & TG(54:5)_TG(18:0/18:2/18:3) & HMDB0045301 & M3 & 0.28 & $0 . c$ \\
\hline Triacylglycerol & TG(54:5)_TG(16:0/18:1/20:4) & HMDB0044098 & M3 & 0.28 & $0 . c$ \\
\hline Triacylglycerol & TG(54:2)_TG(18:0/18:1/18:1) & HMDB0005403 & M3 & -0.41 & $0 . c$ \\
\hline Triacylglycerol & TG(54:6)_TG(16:0/16:1/22:5) & HMDB0044591 & M3 & 0.04 & $0 . c$ \\
\hline Triacylglycerol & TG(54:6)_TG(18:0/18:3/18:3) & HMDB0052887 & M3 & 0.04 & $0 . c$ \\
\hline Triacylglycerol & TG(54:6)_TG(16:0/18:1/20:5) & HMDB0044133 & M3 & 0.04 & $0 . c$ \\
\hline Triacylglycerol & TG(56:5)_TG(18:1/18:1/20:3) & HMDB0049883 & M3 & -0.64 & $0 . C$ \\
\hline Unannoated & X310-1-MZ310RT517 & & M1 & -1.13 & $0 . c$ \\
\hline Unannoated & X310-2-MZ310RT526 & & M1 & -2.61 & $0 . C$ \\
\hline Unannoated & X310-1-MZ310RT307 & & M1 & -1.63 & $0 . c$ \\
\hline Unannoated & X310-2-MZ310RT320 & & M1 & -1.73 & $0 . c$ \\
\hline
\end{tabular}

\section{Metabolite enrichment and Metabolic pathway analysis}

Based on these identified metabolites, metabolic pathway analysis (MetPA) analysis was performed (Fig. 4a, Table 3). In Fig. 4a, -log (p value) and pathway impact were the $X$ and $Y$ axes of the bubble diagram. It could be observed that these metabolites were significantly enriched in metabolic pathways including Glycerophospholipid metabolism, Sphingolipid metabolism, Phenylalanine, tyrosine and tryptophan biosynthesis, Arginine biosynthesis, Histidine metabolism, Ether lipid metabolism. Furthermore, metabolites set enrichment analysis (MSEA) was also performed based on the Metabolites Set in KEGG database (Fig. 4b, Table 4). As shown in Fig. 4b, -log ( $p$ value) and fold change were the $X$ and $Y$ axes of the bubble diagram. MSEA analysis showed that Purine metabolism, Porphyrin and chlorophyll metabolism, Fatty acid degradation, Taurine and hypotaurine metabolism, Phenylalanine metabolism, Phenylalanine, tyrosine and tryptophan biosynthesis, Fatty acid biosynthesis, etc., were involved in metabolic pathways of these metabolites enriched. 
Table 3

The pathways of the differential metabolites enriched

\begin{tabular}{|c|c|c|c|c|}
\hline Pathway name & Match status & p-value & $-\log (p)$ & Impact \\
\hline Glycerophospholipid metabolism & $4 / 36$ & 0.00555 & 5.1945 & 0.26445 \\
\hline Sphingolipid metabolism & $3 / 21$ & 0.00826 & 4.7961 & 0.06896 \\
\hline Arginine biosynthesis & $2 / 14$ & 0.03220 & 3.4358 & 0.11675 \\
\hline Histidine metabolism & $2 / 16$ & 0.04139 & 3.1848 & 0.00000 \\
\hline Ether lipid metabolism & $2 / 20$ & 0.06227 & 2.7763 & 0.14458 \\
\hline Aminoacyl-tRNA biosynthesis & $3 / 48$ & 0.07376 & 2.6069 & 0.00000 \\
\hline Phenylalanine, tyrosine and tryptophan biosynthesis & $1 / 4$ & 0.08013 & 2.5241 & 0.50000 \\
\hline Linoleic acid metabolism & $1 / 5$ & 0.09917 & 2.3109 & 0.00000 \\
\hline Alanine, aspartate and glutamate metabolism & $2 / 28$ & 0.11195 & 2.1897 & 0.42068 \\
\hline Nitrogen metabolism & $1 / 6$ & 0.11783 & 2.1385 & 0.00000 \\
\hline D-Glutamine and D-glutamate metabolism & $1 / 6$ & 0.11783 & 2.1385 & 0.50000 \\
\hline Purine metabolism & $3 / 65$ & 0.14751 & 1.9139 & 0.03102 \\
\hline Ascorbate and aldarate metabolism & $1 / 8$ & 0.15403 & 1.8706 & 0.00000 \\
\hline Taurine and hypotaurine metabolism & $1 / 8$ & 0.15403 & 1.8706 & 0.42857 \\
\hline Phenylalanine metabolism & $1 / 10$ & 0.18879 & 1.6671 & 0.35714 \\
\hline alpha-Linolenic acid metabolism & $1 / 13$ & 0.23835 & 1.4340 & 0.00000 \\
\hline Glycosylphosphatidylinositol (GPI)-anchor biosynthesis & $1 / 14$ & 0.25420 & 1.3696 & 0.00399 \\
\hline Butanoate metabolism & $1 / 15$ & 0.26974 & 1.3103 & 0.00000 \\
\hline Nicotinate and nicotinamide metabolism & $1 / 15$ & 0.26974 & 1.3103 & 0.00000 \\
\hline Glycerolipid metabolism & $1 / 16$ & 0.28496 & 1.2554 & 0.01402 \\
\hline Pantothenate and CoA biosynthesis & $1 / 19$ & 0.32881 & 1.1123 & 0.00000 \\
\hline beta-Alanine metabolism & $1 / 21$ & 0.35659 & 1.0312 & 0.00000 \\
\hline Pyruvate metabolism & $1 / 22$ & 0.37005 & 0.9941 & 0.08398 \\
\hline Glutathione metabolism & $1 / 28$ & 0.44529 & 0.8090 & 0.01966 \\
\hline Porphyrin and chlorophyll metabolism & $1 / 30$ & 0.46838 & 0.7585 & 0.00000 \\
\hline Glyoxylate and dicarboxylate metabolism & $1 / 32$ & 0.49054 & 0.7123 & 0.00000 \\
\hline Cysteine and methionine metabolism & $1 / 33$ & 0.50128 & 0.6906 & 0.00000 \\
\hline Arachidonic acid metabolism & $1 / 36$ & 0.53220 & 0.6307 & 0.00000 \\
\hline Arginine and proline metabolism & $1 / 38$ & 0.55177 & 0.5946 & 0.08600 \\
\hline Fatty acid degradation & $1 / 39$ & 0.56126 & 0.5776 & 0.00000 \\
\hline Steroid biosynthesis & $1 / 42$ & 0.58856 & 0.5301 & 0.00000 \\
\hline Primary bile acid biosynthesis & $1 / 46$ & 0.62242 & 0.4741 & 0.00758 \\
\hline
\end{tabular}


Table 4

MSEA pathway analysis of the differential metabolites enriched

\begin{tabular}{|c|c|c|c|}
\hline Metabolite Set & Total & Hits & FDR \\
\hline Purine metabolism & 65 & 4 & 0.000000 \\
\hline Porphyrin and chlorophyll metabolism & 30 & 3 & 0.000186 \\
\hline Taurine and hypotaurine metabolism & 8 & 1 & 0.016056 \\
\hline Fatty acid degradation & 39 & 1 & 0.040924 \\
\hline Phenylalanine metabolism & 10 & 2 & 0.044818 \\
\hline Phenylalanine, tyrosine and tryptophan biosynthesis & 4 & 2 & 0.044818 \\
\hline Fatty acid biosynthesis & 47 & 1 & 0.070722 \\
\hline Primary bile acid biosynthesis & 46 & 3 & 0.040924 \\
\hline Cysteine and methionine metabolism & 33 & 3 & 0.050999 \\
\hline Pentose and glucuronate interconversions & 18 & 1 & 0.205370 \\
\hline Valine, leucine and isoleucine degradation & 40 & 3 & 0.205370 \\
\hline Sphingolipid metabolism & 21 & 5 & 0.205370 \\
\hline Glutathione metabolism & 28 & 2 & 0.205370 \\
\hline Arginine biosynthesis & 14 & 3 & 0.205370 \\
\hline Valine, leucine and isoleucine biosynthesis & 8 & 4 & 0.208410 \\
\hline Arginine and proline metabolism & 38 & 4 & 0.205370 \\
\hline beta-Alanine metabolism & 21 & 2 & 0.249570 \\
\hline Steroid hormone biosynthesis & 85 & 3 & 0.252260 \\
\hline Selenocompound metabolism & 20 & 1 & 0.480370 \\
\hline Aminoacyl-tRNA biosynthesis & 48 & 17 & 0.443750 \\
\hline Caffeine metabolism & 10 & 2 & 0.480370 \\
\hline Pantothenate and CoA biosynthesis & 19 & 1 & 0.534600 \\
\hline Alanine, aspartate and glutamate metabolism & 28 & 3 & 0.648530 \\
\hline alpha-Linolenic acid metabolism & 13 & 1 & 0.648530 \\
\hline Tryptophan metabolism & 41 & 4 & 0.780710 \\
\hline Ubiquinone and other terpenoid-quinone biosynthesis & 9 & 1 & 0.649670 \\
\hline Tyrosine metabolism & 42 & 1 & 0.649670 \\
\hline Glycine, serine and threonine metabolism & 33 & 5 & 0.863790 \\
\hline Glyoxylate and dicarboxylate metabolism & 32 & 3 & 0.803010 \\
\hline Pyrimidine metabolism & 39 & 2 & 0.803010 \\
\hline Glycerophospholipid metabolism & 36 & 1 & 0.788010 \\
\hline Butanoate metabolism & 15 & 1 & 0.803010 \\
\hline Pentose phosphate pathway & 22 & 1 & 0.803010 \\
\hline D-Glutamine and D-glutamate metabolism & 6 & 1 & 0.824110 \\
\hline Nitrogen metabolism & 6 & 1 & 0.824110 \\
\hline Lysine degradation & 25 & 2 & 0.943180 \\
\hline Biotin metabolism & 10 & 1 & 0.869120 \\
\hline Nicotinate and nicotinamide metabolism & 15 & 1 & 0.943180 \\
\hline Histidine metabolism & 16 & 1 & 0.959140 \\
\hline Galactose metabolism & 27 & 1 & 0.959140 \\
\hline Glycerolipid metabolism & 16 & 1 & 0.959140 \\
\hline
\end{tabular}

Page $11 / 18$ 


\section{ROC curves of significant metabolotics in PCOS patients and controls}

In order to further distinguish PCOS from controls, ROC curves analysis was also conducted on these changed metabolites, the top 10 metabolites of highest AUC and their intensity in PCOS and controls were presented in Fig. 5. These metabolites were Leu-Ala/lle-Ala (AUC = 1.00), 3-(4-Hydroxyphenyl)propionic acid $(A U C=0.998)$, lle-Val/Leu-Val (AUC = 0.982), Gly-Val/Val-Gly (AUC = 0.982), aspartic acid (AUC = 0.968), DG(34:2)_DG(16:0/18:2) (AUC=0.951), DG(34:1)_DG(16:0/18:1) (AUC=0.938), Phe-Trp (AUC=0.935), DG(36:1)_DG(18:0/18:1) (AUC=0.905), Leu-Leu/Leu-lle (AUC=0.904).

\section{Discussion}

PCOS is a kind of common endocrine syndrome as well as metabolic disorder, which harms the reproductive system and the overall body metabolism of the patients [2]. In this study, we utilized metabolomics approach by UPLC-HRMS technology to study the metabolic changes of in 31 PCOS patients and 31 healthy controls. The metabolomics analysis showed that in PCOS patients serum, there were 146 significantly varied metabolites, among them 68 were downregulated, 78 were upregulated. These metabolites mainly belong to Triacylglycerols, Glycerophosphocholines, Acylcarnitines, Diacylglycerols, Peptides, Amino acids, Glycerophosphoethanolamines and FA. Pathway analysis showed that these metabolites were enriched in pathways including Glycerophospholipid metabolism, Fatty acid degradation, Fatty acid biosynthesis, Ether lipid metabolism etc. Diagnosis value assessment by ROC analysis showed that the changed metabolites including Leu-Ala/Ile-Ala, 3-(4-Hydroxyphenyl) propionic acid, Ile-Val/Leu-Val, Gly-Val/Val-Gly, aspartic acid, DG(34:2)_DG(16:0/18:2), DG(34:1)_DG(16:0/18:1), Phe-Trp, DG(36:1)_DG(18:0/18:1), Leu-Leu/Leu-Ile had higher AUC values, indicated significant role in PCOS.

Genomic, proteomic, and metabolomic approaches have been introduced to various diseases research. Metabolomics enables to identify both the endogenous metabolites from downstream output of the genome and the exogenous metabolites from the upstream input from the environment, therefore allowing researchers to explore the nexus of gene-environment interactions and providing unique insights into the fundamental causes of disease [10,12]. To date, many metabolomic studies in PCOS have revealed the matabolic profiles and changes in PCOS patients under various conditions. In Zhang's study, they recruited 286 subjects and grouped into healthy women (C), those with hyperandrogenism (B1), those with IR but not obese (B2) and obese women with IR (B3) in PCOS in order to reveal metabolic profiling for women with hyperandrogenism and IR in PCOS ${ }^{[13}$ ] . The serum metabolomics analysis identified 59 differential metabolites, including palmitic acid, cholesterol, myo-inositol, D-allose, 1,5-anhydro-D-sorbitol, 1-monopalmitin, 1-monostearin, glycerol 1phosphate, malic acid and citric acid, and these differential metabolites were related to biosynthesis of unsaturated fatty acids, and citrate cycle. Another study enrolled 10 PCOS patients and 10 healthy people and identified six biomarkers, L-Carnitine, LPE (22:5), Sphinganine, LPC (18:2), DHEAS and Glycocholic acid, these biomarkers belonged to metabolic pathway including lipid metabolism, carnitine metabolism, androgen metabolism, and bile acid metabolism [14]. Zhao's serum metabolomics study of PCOS found Significant increase in the levels of unsaturated free fatty acids, fatty acid amides, sulfated steroids, glycated amino acid and the decrease in levels of lysophosphatidylcholines, lysophosphatidylethanolamines, and free fatty acid (FFA) 18:1/FFA 18:0, FFA 20:3, dihydrotestosterone sulfate, glycated phenylalanine, and uridine were combined as a diagnostic biomarker for PCOS [15]. Murri et al reviewed the the metabolites and metabolic pathways associated with PCOS according to recent metabolomic studies, and reported that PCOS-associated metabolites were involved mostly in carbohydrate, fat, and protein metabolism [16]. These studies revealed the metabolomic changes in PCOS patients, offered new insights into disease processes, and the discriminated metabolites may also help to provide reference for PCOS diagnosis and therapy. But limited sample sizes and the different metabolomic techniques used impose important limitations when aiming to integrate the results of the different studies conducted to date.

As well acknowledged that, PCOS is a kind of metabolic disorders, IR and thereby induced obesity are common symptoms in PCOS patients. Hence, lipid and liprotein metabolic abnormalities are accompanied with the PCOS progression [17]. In present study, for the 146 identified differential metabolites, there were 36 metabolites belong to Triacylglycerols, and the identified 36 metabolites were all upregulated in PCos group. Triacylglycerols, also named as triglyceride (TG), are the main components of oils and fats. Overweight PCOS patients usually have lipid abnormalities, including higher level of serum TG. Cross sectional study on PCOS showed that subjects with PCOS demonstrated higher waist:hip ratio (WHR), T, TG, VLDL-cholesterol concentrations ( $P<0.05)$ [18]. Another study found that in PCOS women, there is a significant decrease TG following VitD supplementation for 8 weeks [19]. In addition, fatty acids were also included in the identified differential metabolites, the three FAs (Dihomo-alpha-linolenic acid, Myristoleic acid isomer 1, Myristoleic acid isomer 2) were all downregulated in PCOS group. But for the three FAs identified in this study, the previous publications about them in PCOS are limited. Dihomo-alpha-linolenic acid is a rare polyunsaturated fatty acid of the $\omega-3$ series, it accounts a small portion of the serum phospholipid fatty acids in normal humans but have significant role. PUFA could modulate hormonal and lipid profiles of the body, lowered TG and cholesterol levels, but patients with PCOS usually showe abnormal levels of PUFA metabolites. Study focused on differences in FA profiles of abdominal subcutaneous adipose tissue between pregnant women with and without PCOS found that total monounsaturated FA (MUFA) $(p<0.0004)$ were higher in PCOS women than non-PCOS women, but total polyunsaturated FA (PUFA) was lower in PCOS than non-PCOS women $(p<0.004)$ [20]. $\omega-3$ PUFA supplementation has the positive modulation effect on the PCOS ovarian function, and potentiates the cellular development and steroid biosynthesis in PCOS [21]. Animal model study also showed that $\omega-3$ PUFA had effective role in improving lipid and hormonal profile, reducing blood glucose, weight gain and histopathological damages in PCOS rats [22]

As aforementioned, PCOS-associated metabolites were involved mostly in lipid and liprotein metabolic abnormalities. In present study, pathway analysis found that these identified differential metabolites were associated with the various pathways, especially including Glycerophospholipid metabolism, Sphingolipid metabolism, Phenylalanine metabolism, Ether lipid metabolism, Purine metabolism, Fatty acid degradation, Fatty acid biosynthesis, etc. Untargeted metabolomics approach on PCOS follicular fluid found significant abundance differences of glycerolipid, glycerophospholipids, sphingolipids, and carboxylic acids compared with healthy women, and these metabolism dysfunctions are contributed to decline the 2 pronuclei (PN) fertilization rate during IVF procedure [23]. Another LC-MS-based metabolomics showed that abnormalities of glycerophospholipid, glycerolipid and FA metabolisms were involved in the pathogenesis of PCOS and IR complications [24]. Amino acid metabolism is also a critical metabolism pathway of the body. In this study, except for the

Page $12 / 18$ 
identification of eight differential amino acids in PCOS, several related amino acid pathway were also identified, indicating the involvement of amino acid metabolism in PCOS., Zhao et al found that, in PCOS patients, the levels of phenylalanine, tyrosine and tryptophan are generally increased, and the ovulatory dysfunction of PCOS patients was associated with raised production of serine, threonine, phenylalanine, tyrosine and ornithine [25]. Fatty acid related pathways, including fatty acid degradation and biosynthesis were also found to associated with the changed metabolites in PCOS of this study. And this was corresponded to the differential metabolites in PCOS compared to the healthy controls.

\section{Conclusion}

In conclusion, in this study, we utilized metabolomics approach by UPLC-HRMS technology to study the metabolic changes of in 31 PCOS patients and 31 healthy controls. The metabolomics analysis showed that in PCOS patients serum, there were 146 significantly varied metabolites, among them 68 were downregulated, 78 were upregulated. These metabolites mainly belong to Triacylglycerols, Glycerophosphocholines, Acylcarnitines, Diacylglycerols, Peptides, Amino acids, Glycerophosphoethanolamines and FA. Pathway analysis showed that these metabolites were enriched in pathways including Glycerophospholipid metabolism, Fatty acid degradation, Fatty acid biosynthesis, Ether lipid metabolism etc. Diagnosis value assessment by ROC analysis showed that the changed metabolites including Leu-Ala/Ile-Ala, 3-(4-Hydroxyphenyl) propionic acid, Ile-Val/Leu-Val, Gly-Val/Val-Gly, aspartic acid, DG(34:2)_DG(16:0/18:2), DG(34:1)_DG(16:0/18:1), Phe-Trp, DG(36:1)_DG(18:0/18:1), Leu-Leu/Leu-Ile had higher AUC values, indicated significant role in PCOS.

\section{Declarations}

\section{Acknowledgement:}

None.

\section{Funding:}

None.

\section{Conpeting interest:}

The authors declare that they have no conflict of interest.

\section{Availability of data and materials}

Data sharing is not applicable to this article as no datasets were generated or analysed during the current study.

\section{Conflict of publication:}

Not applicable.

\section{Authors' contributions}

Conception and design of the research: $\mathrm{HXX} \square \mathrm{Y} Y$

Acquisition of data: PL T, RY Q

Analysis and interpretation of data: ZC Z

Statistical analysis: ZJ W

Drafting the manuscript: LC Z

Revision of manuscript for important intellectual content: ZJ W

All authors read and approved the final manuscript

\section{Ethics approval and consent to participate:}

The study was approved by the Ethics Committee of Zhejiang Provincial Hospital of Chinese Medicine. [2020-KL-155-02]. All the participators signed informed consent forms before the start of the study. All protocols are carried out in accordance with relevant guidelines and regulations of "The Regulations of Ethical Reviews of Biomedical Research Involving Human Subjects". 


\section{References}

1. Empowering women with PCOS. Lancet Diabetes Endocrinol. 2019 10;7(10):737.

2. Escobar-Morreale HF. Polycystic ovary syndrome: definition, aetiology, diagnosis and treatment. Nat Rev Endocrinol. 2018;14(5):270-284.

3. Zawadzki J, Duniaf A. Diagnostic criteria for polycystic ovary syndrome: Towards a rational approach. Boston: Blackwell Scientific Publications. 1992.

4. Rotterdam ESHRE/ASRM-Sponsored PCOS consensus workshop group. Revised 2003 consensus on diagnostic criteria and long-term health risks related to polycystic ovary syndrome (PCOS). Hum. Reprod. 2004;19 (1):41-47.

5. Azziz R, Carmina E, Dewailly D, Diamanti-Kandarakis E, Escobar-Morreale HF, Futterweit W, et al. Positions statement: Criteria for defining polycystic ovary syndrome as a predominantly hyperandrogenic syndrome: An androgen excess society guideline. J Clin Endocrinol Metab. 2006;91:4237-45.

6. Cobin RH. Cardiovascular and metabolic risks associated with PCOS. Intern Emerg Med. 2013 Apr;8 Suppl 1:S61-4.

7. Kakoly NS, Moran LJ, Teede HJ, Joham AE. Cardiometabolic risks in PCOS: a review of the current state of knowledge. Expert Rev Endocrinol Metab. 2019;14(1):23-33.

8. Peigné M, Dewailly D. Long term complications of polycystic ovary syndrome (PCOS). Ann Endocrinol (Paris). 2014 Sep;75(4):194-9.

9. Chang AY, Lalia AZ, Jenkins GD, et al. Combining a Nontargeted and Targeted Metabolomics Approach to Identify Metabolic Pathways Significantly Altered in Polycystic Ovary Syndrome. Metabolism. 2017;71:52-63.

10. Wishart DS. Emerging applications of metabolomics in drug discovery and precision medicine. Nat Rev Drug Discov. 2016;15(7):473-84.

11. Buszewska-Forajta M, Rachoń D, Stefaniak A, et al. Identification of the metabolic fingerprints in women with polycystic ovary syndrome using the multiplatform metabolomics technique. J Steroid Biochem Mol Biol. 2019;186:176-184.

12. Houten SM. Metabolomics: unraveling the chemical individuality of common human diseases. Ann Med. 2009;41:402-407.

13. Zhang Z, Hong Y, Chen M, et al. Serum metabolomics reveals metabolic profiling for women with hyperandrogenism and insulin resistance in polycystic ovary syndrome. Metabolomics. 2020;16(2):20.

14. Jia C, Xu H, Xu Y, Shi Q. Serum metabolomics analysis of patients with polycystic ovary syndrome by mass spectrometry. Mol Reprod Dev. 2019 03;86(3):292-297.

15. Zhao X, Xu F, Qi B, et al. Serum Metabolomics Study of Polycystic Ovary Syndrome Based on Liquid Chromatography - Mass Spectrometry. J Proteome Res. 2014;13(2):1101-11.

16. Murri M, Insenser M, Escobar-Morreale HF. Metabolomics in polycystic ovary syndrome. Clin Chim Acta. 2014;429:181-8.

17. Diamanti-Kandarakis E, Papavassiliou AG, Kandarakis SA, Chrousos GP. Pathophysiology and types of dyslipidemia in PCoS. Trends Endocrinol Metab. 2007;18(7):280-5.

18. Pirwany IR, Fleming R, Greer IA, Packard CJ Sattar N. Lipids and lipoprotein subfractions in women with PCOS: relationship to metabolic and endocrine parameters. Clin Endocrinol (Oxf). 2001 Apr;54(4):447-53.

19. Irani M, Seifer DB, Grazi RV, Irani S, Rosenwaks Z, Tal R. Vitamin D Decreases Serum VEGF Correlating with Clinical Improvement in Vitamin D-Deficient Women with PCOS: A Randomized Placebo-Controlled Trial. Nutrients. 2017;9(4).

20. Emami N, Alizadeh A, Moini A, Yaghmaei P, Shahhosseini M. Differences in fatty acid profiles and desaturation indices of abdominal subcutaneous adipose tissue between pregnant women with and without PCOS. Adipocyte. 2020 12;9(1):16-23.

21. Hu X, Weng X, Tian Y, et al. Effects of omega-3 polyunsaturated fatty acids on steroidogenesis and cellular development in PCOS rats. Food Funct. 2019 May 22;10(5):2504-2514.

22. Komal F, Khan MK, Imran M, et al. Impact of different omega-3 fatty acid sources on lipid, hormonal, blood glucose, weight gain and histopathological damages profile in PCOS rat model. J TransI Med. 202009 14;18(1):349.

23. Liu L, Yin TL, Chen Y, et al. Follicular dynamics of glycerophospholipid and sphingolipid metabolisms in polycystic ovary syndrome patients. J Steroid Biochem Mol Biol. 2019 01;185:142-149.

24. Chen YX, Zhang XJ, Huang J, et al. UHPLC/Q-TOFMS-based plasma metabolomics of polycystic ovary syndrome patients with and without insulin resistance. J Pharm Biomed Anal. 2016 Mar 20;121:141-150.

25. Zhao Y, Fu L, Li R, et al. Metabolic profiles characterizing different phenotypes of polycystic ovary syndrome: plasma metabolomics analysis. BMC Med. 2012 Nov 30;10:153.

\section{Figures}



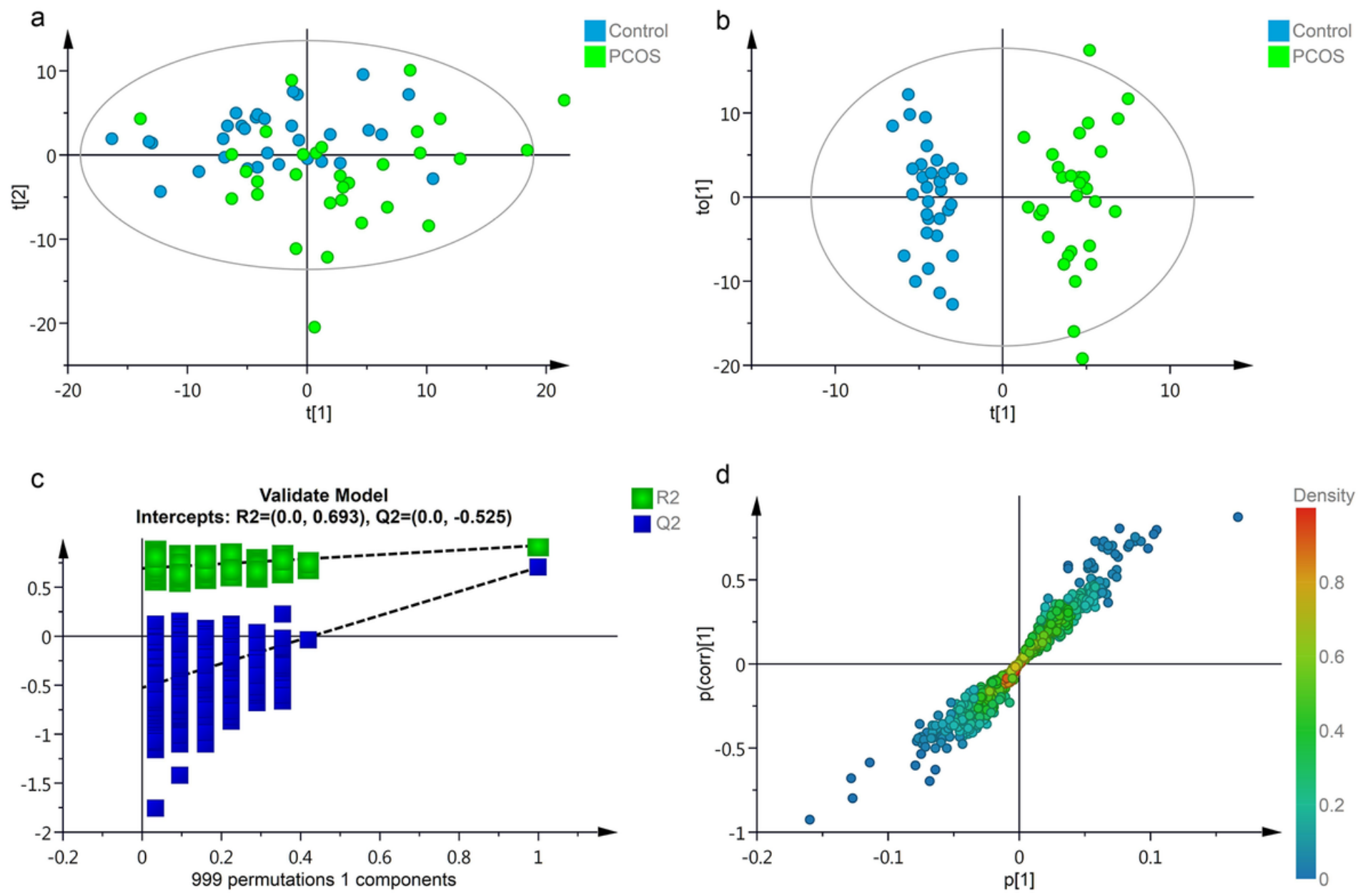

\section{Figure 1}

Multivariate statistical analysis of the serum metabolites in the study subjects. a: unsupervised PCA score plots of metabolic phenotypes between PCOS and CON groups. Metabolomics data was log transformed and scaled to unit variance for modeling. Model parameter: R2X $=0.86$ (cumulative variance proportion of 9 principal components). b: Score plot of OPLS-DA modeling to maximize inter-group differentiation of metabolomic data between PCOS and CON groups. Model parameter: 1 predictive component +2 orthogonal component, R2Y=0.93, Q2=0.70. c: 999 times permutation test result of OPLS-DA modeling. d: S-plot of OPLS-DA modeling. 

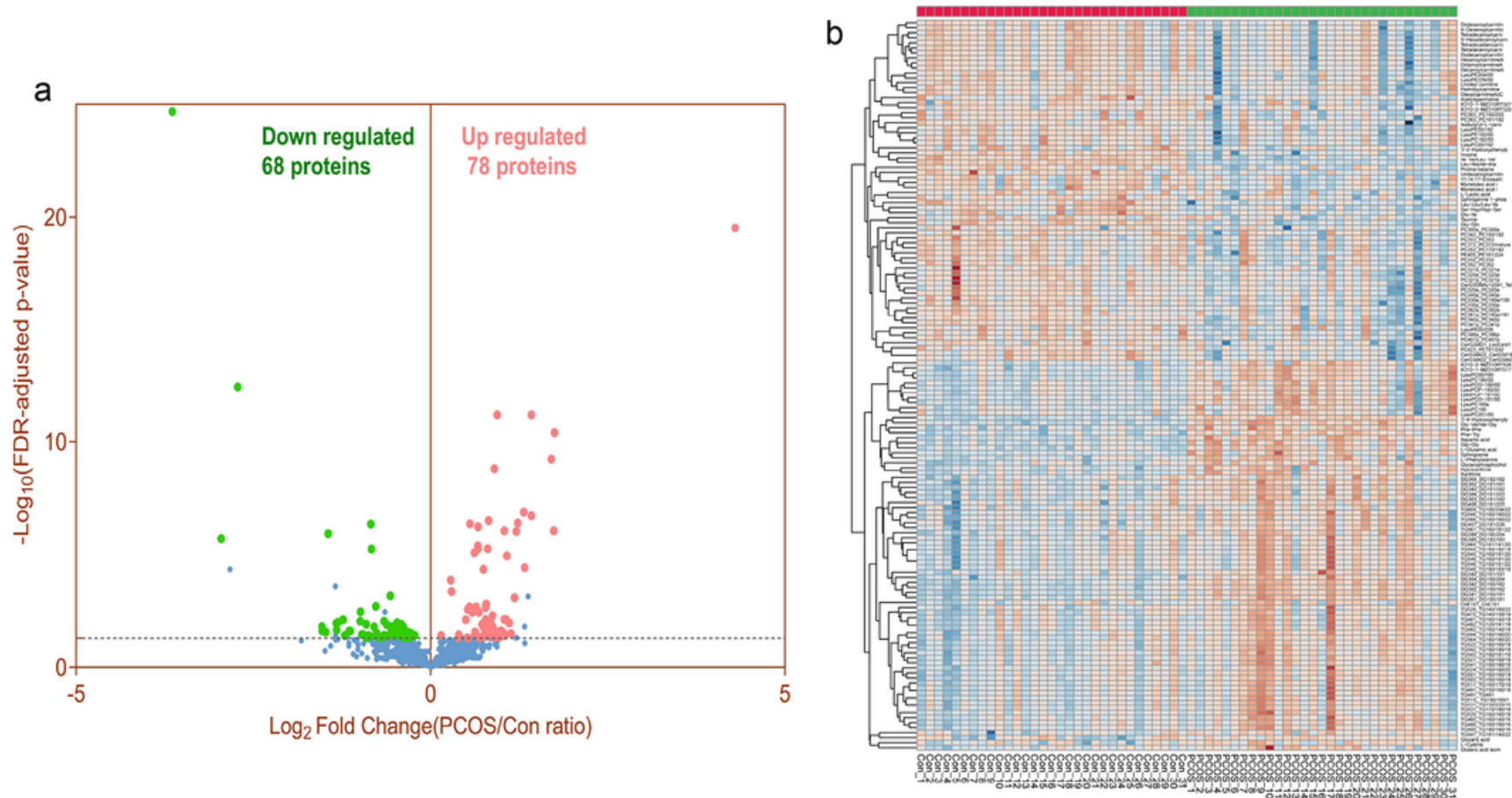

\section{Figure 2}

Identification of significant metabolotics by UPLC-HRMS. a: Volcano plot to visualize differential metabolites of significance between PCOS and CON group. Metabolites with FDR adjusted p-value $<=0.05$ were highlighted with red (up-regulated) and green color (down-regulated), respectively. b: Clustering analysis result using differential metabolites of significance in Student's t-test analysis (FDR adjusted p-value < 0.05).

PCOS vs. Control差异代谢物数量

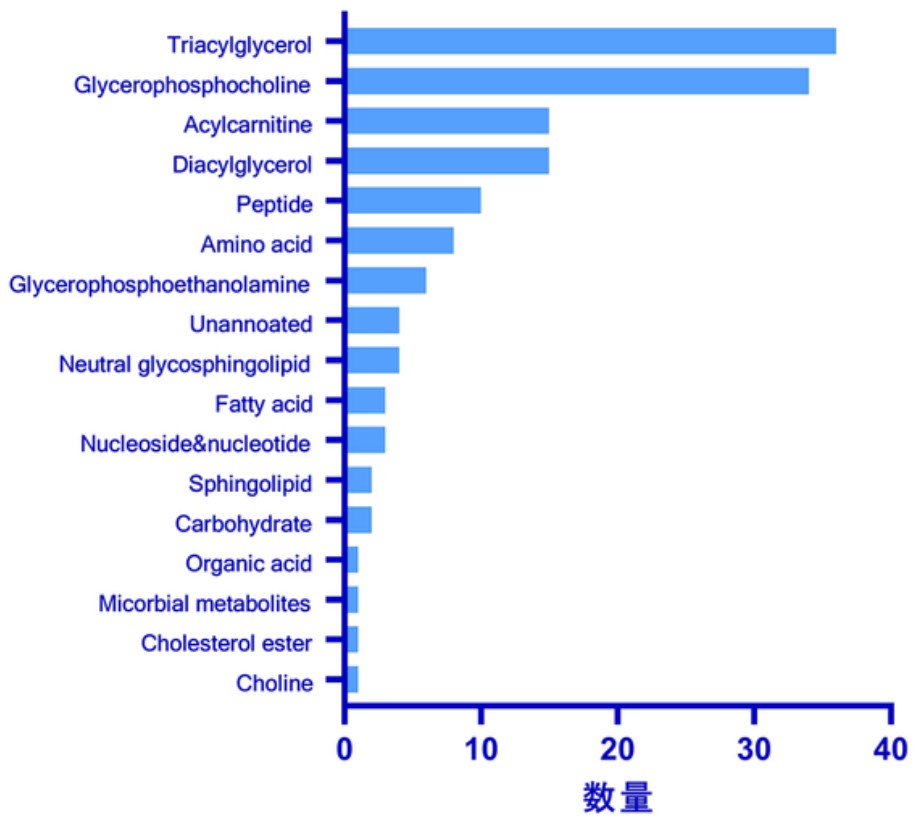

PCOS vs. Control差异代谢物变化比例

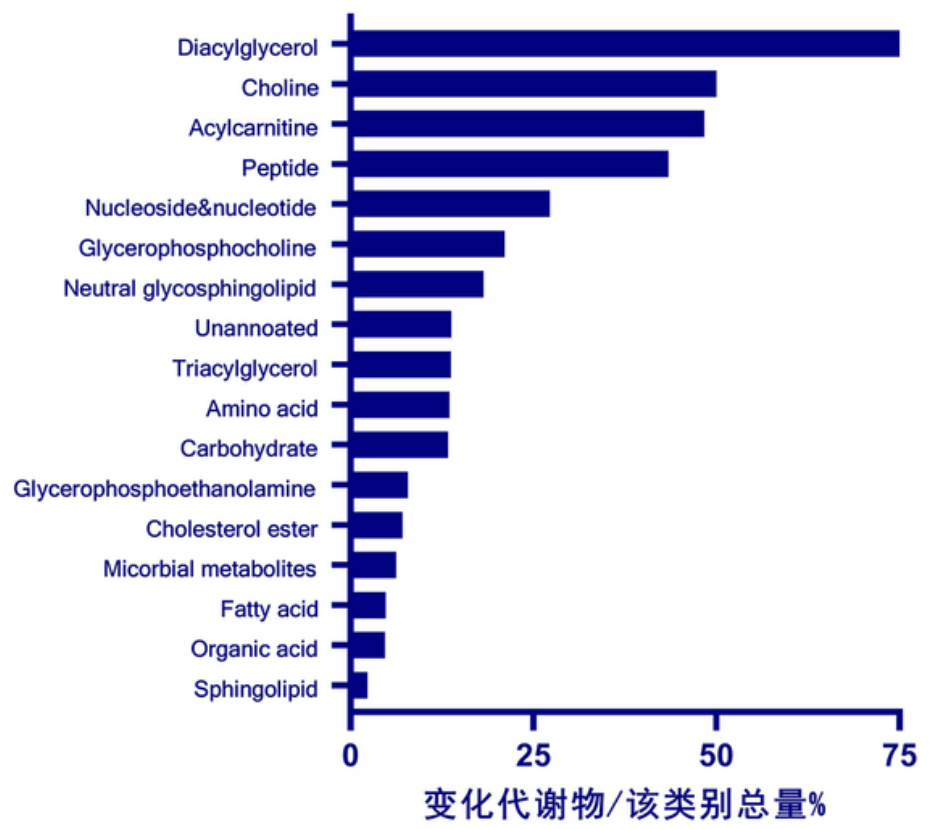

Figure 3

Chemical structure classification of differential metabolites between PCOS and control group. 


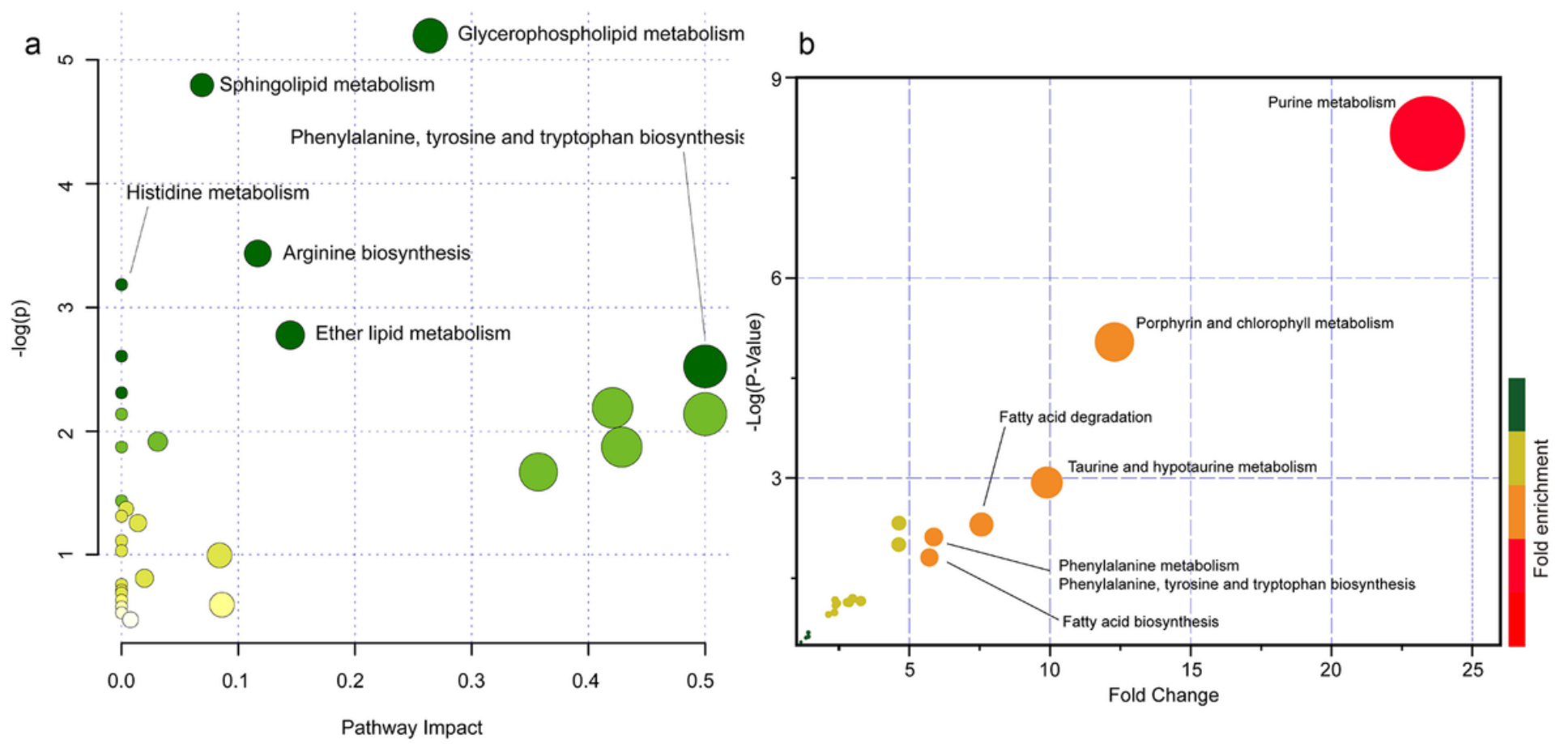

Figure 4

Pathway analysis of the differential metabolites between PCOS vs.CON group. a: Pathway analysis result of differential metabolites between PCOS vs. control group using over-representation method in MetaboAnalyst website (pvalue $<0.05$ of t-test after FDR adjusting). Hypergeometric test and Relativebetweeness centrality algorithm was used for pathway topology analysis, human KEGG pathway library was used. b: Metabolites set enrichment analysis of all metabolites with HMBD identifier using quantitative enrichment analysis method. Pathway-associated metabolite sets (KEGG) containing 84 metabolite sets based on normal human metabolic pathways was used for this MSEA. 
a

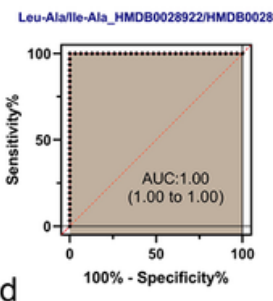

Gly-Valval-Gly_HMOB0028854/HMOBO02912

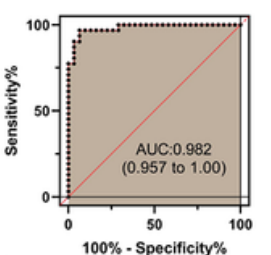

g

DG(34:1)_DG(16:0/18:11__HMDB0007101

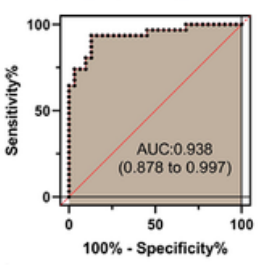

j

Leu-Leu/Leu-lle_HMDB0028933

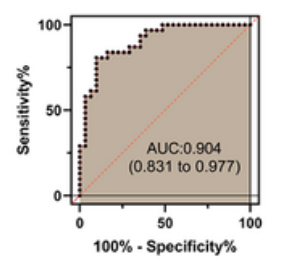

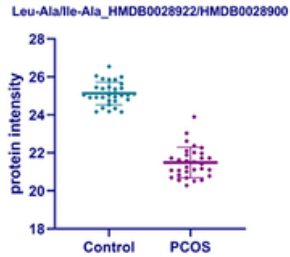

Gly-ValNal-Gly_HMOB0028854/HMOB0029127
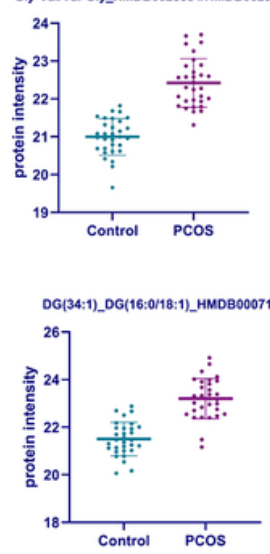

Leu-Leu/Leu-Ile_HMDB0028933

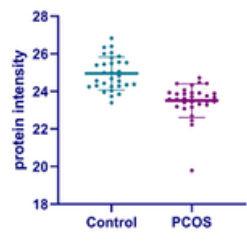

b

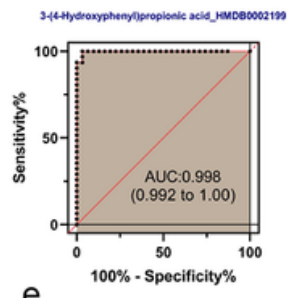

e

Aspartic acid_HMDB0000191

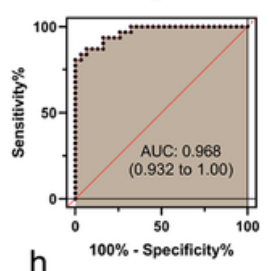

$\mathrm{h}$

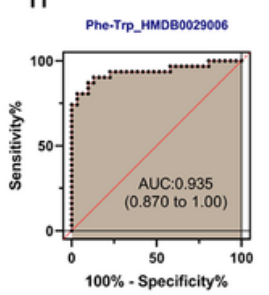

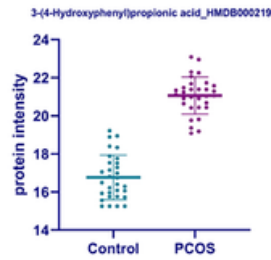

Aspartic acid_HMDB0000191
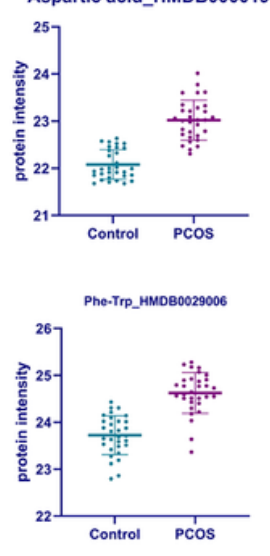

C

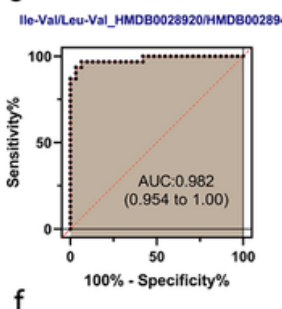

DG(36:2)_DG(16:0/18:2)_нMDBB0007103

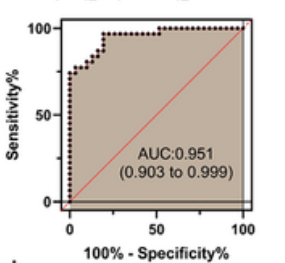

i

DG(36:1)_DG(18:0/18:1)_HMDB0007159

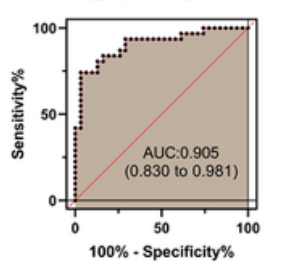

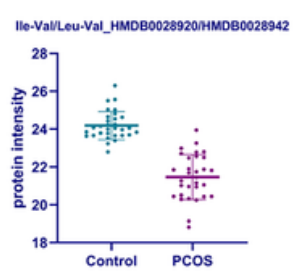

DG(34:2)_DG(16:0/18:2)_HMDB0007103

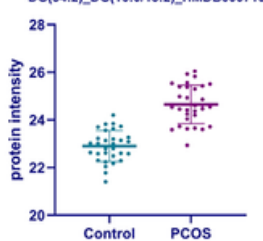

DG(36:1)_OG(18:0/18:1)_HMOB0007159

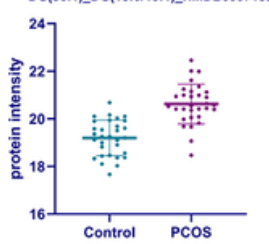

Figure 5

ROC-curve analysis of top 10 metabolites ranked AUC values. 